\title{
Bioremediation: An Overview on Current Practices, Advances, and New Perspectives in Environmental Pollution Treatment
}

\author{
Raluca Maria Hlihor, ${ }^{1,2}$ Maria Gavrilescu, ${ }^{2,3}$ Teresa Tavares, ${ }^{4}$ \\ Lidia Favier, ${ }^{5}$ and Giuseppe Olivieri ${ }^{6,7}$ \\ ${ }^{1}$ Department of Horticultural Technologies, Faculty of Horticulture, "Ion Ionescu de la Brad" University of Agricultural Sciences and \\ Veterinary Medicine, 3 Mihail Sadoveanu Alley, 700490 Iași, Romania \\ ${ }^{2}$ Department of Environmental Engineering and Management, Faculty of Chemical Engineering and Environmental Protection, \\ "Gheorghe Asachi” Technical University of Iaşi, 73 Prof. Dr. Docent D. Mangeron Street, 700050 Iaşi, Romania \\ ${ }^{3}$ Academy of Romanian Scientists, 54 Splaiul Independentei, 050094 Bucharest, Romania \\ ${ }^{4}$ Centre of Biological Engineering (CEB), University of Minho, Campus de Gualtar, 4710-057 Braga, Portugal \\ ${ }^{5}$ Ecole Nationale Supérieure de Chimie de Rennes, CNRS, UMR 6226, 11 Allée de Beaulieu, CS 50837, 35708 Rennes Cedex 7, France \\ ${ }^{6}$ Department of Chemical, Materials and Industrial Production Engineering, Università degli Studi di Napoli Federico II, \\ Piazzale V. Tecchio 80, 80125 Napoli, Italy \\ ${ }^{7}$ Bioprocess Engineering Group, Wageningen University and Research, Droevendaalsesteeg 1, 6708 AA Wageningen, Netherlands
}

Correspondence should be addressed to Raluca Maria Hlihor; raluca.hlihor@tuiasi.ro

Received 7 October 2017; Accepted 10 October 2017; Published 1 November 2017

Copyright ( 2017 Raluca Maria Hlihor et al. This is an open access article distributed under the Creative Commons Attribution License, which permits unrestricted use, distribution, and reproduction in any medium, provided the original work is properly cited.

Environmental pollution generated the need to search for new environmentally friendly, low-cost, and more efficient environmental clean-up techniques for its removal or reduction. Bioremediation, a branch of environmental biotechnology, is nowadays considered as one of the most promising alternatives. This technology uses the amazing ability of microorganisms or plants to accumulate, detoxify, degrade, or remove environmental contaminants. Bioremediation provides the transformation and/or even removal of organic and inorganic pollutants, even when they are present at low concentration. Continuous efforts are still made to understand the mechanisms by which microorganisms and plants remove or transform environmental pollutants. Thus, the purpose of this special issue was to explore different visions on bioremediation, while addressing recent advances and new ideas in the perspective of efficient process scale-up in view of application at larger scales.

Authors' contributions cover various topics with a range of papers including original research and review articles spanning studies in remediation of different environments which outline new findings in the biotechnology field. This special issue contains five papers including one review article and four original research articles. A brief description of these five manuscripts is detailed below.

During the treatment of wastewater with high ammonium concentrations, as is the effluent originating from anaerobic digestion of pig slurry, the presence of free ammonia $\left(\mathrm{NH}_{3}\right.$ or $\left.\mathrm{FA}\right)$ and/or free nitrous acid $\left(\mathrm{HNO}_{2}\right.$ or FNA) can affect the performance of the partial nitrification process. Thus, in the paper titled "Effect of Free Ammonia, Free Nitrous Acid, and Alkalinity on the Partial Nitrification of Pretreated Pig Slurry, Using an Alternating Oxic/Anoxic SBR" by M. Belmonte et al., the authors applied a strategy allowing the use of organic matter to partially remove nitrite $\left(\mathrm{NO}_{2}{ }^{-}\right)$and nitrate $\left(\mathrm{NO}_{3}{ }^{-}\right)$generated during oxic phases. Stable partial nitrification was achieved during the treatment of the effluent of an anaerobic reactor fed with pig slurry.

In the paper titled "Identification of Multiple Dehalogenase Genes Involved in Tetrachloroethene-to-Ethene Dechlorination in a Dehalococcoides-Dominated Enrichment Culture," M. Ismaeil et al. investigated a Dehalococcoidesdominated enrichment culture (designated "YN3") that 
dechlorinates tetrachloroethene (PCE) to nontoxic ethene (ETH) with high dechlorination activity. The metagenome of YN3 harbored 18 rdhA genes (designated YN3rdhA1-18) encoding the catalytic subunit of reductive dehalogenase (rdhA), four of which were suggested to be involved in PCE-to-ETH dechlorination based on significant increases in their transcription in response to CE addition. Moreover, metagenome data indicated the presence of three coexisting bacterial species, including novel species of the genus Bacteroides, which might promote CE dechlorination by Dehalococcoides.

Thirty-one mercury-resistant bacterial strains were isolated from the effluent discharge sites of the SIPCOT industrial area in the paper of K. Saranya et al. titled "Bioremediation of Mercury by Vibrio fluvialis Screened from Industrial Effluents." An interesting outcome of this study was that the strain $V$. fluvialis demonstrated, on one hand, a high bioremediation efficiency in the detoxification of mercury from mobile solutions and, on the other hand, a low resistance against antibiotics. Hence, V. fluvialis can be successfully applied as a strain for the ecofriendly removal of mercury.

In the paper titled "Effect of Hydraulic Retention Time on Anaerobic Digestion of Wheat Straw in the Semicontinuous Continuous Stirred-Tank Reactors," X.-S. Shi et al. selected a range of process parameters such as the biogas production, methane content, $\mathrm{pH}$ value, and volatile fatty acids (VFAs) component and demonstrate their influence on Hydraulic Retention Time (HRT) in two operation modes of STR (Stirred-Tank Reactors). In addition, the degradation of cellulose, hemicellulose, and crystalline cellulose in digested wheat straw was also investigated. The obtained results indicated that HRT is an important parameter that affects the performance and stability in the anaerobic digestion of wheat straw.

Recent approaches using low sulfidogenic bioreactors to both remediate and selectively recover metal sulfides from acidic mine drainage are reviewed in the paper of I. Nancucheo et al. The manuscript titled "Recent Developments for Remediating Acidic Mine Waters using Sulfidogenic Bacteria" also highlights the efficiency and drawbacks of these types of treatments for metal recovery and points to future research for enhancing the use of novel acidophilic and acidtolerant sulfidogenic microorganisms in AMD treatment.

We hope that this collection of papers provides to the readers a valuable scientific source and support addressing current practices, advances, and new perspectives applicable in the treatment of environmental pollution and we hope it can also help specialists in the field of biotechnology towards sustainable scale-up.

\section{Acknowledgments}

We would like to extend our gratitude to all the authors who submitted their work for consideration in our special issue and to reviewers for their critical feedback. Contributions of Raluca Maria Hlihor and Maria Gavrilescu to this special issue were supported by a grant of the Romanian National Authority for Scientific Research, CNCS-UEFISCDI (Project no. PN-III-P4-ID-PCE-2016-0683, Contract no. 65/2017).
Teresa Tavares' contribution is supported by the Portuguese Foundation for Science and Technology (FCT) under the scope of the research project PTDC/AAG-TEC/5269/2014, the strategic funding of UID/BIO/04469/2013 unit and COMPETE 2020 (POCI-01-0145-FEDER-006684), and BioTecNorte operation (NORTE-01-0145-FEDER-000004) funded by the European Regional Development Fund under the scope of NORTE 2020 (Programa Operacional Regional do Norte).
Raluca Maria Hlihor
Maria Gavrilescu
Teresa Tavares
Lidia Favier
Giuseppe Olivieri 

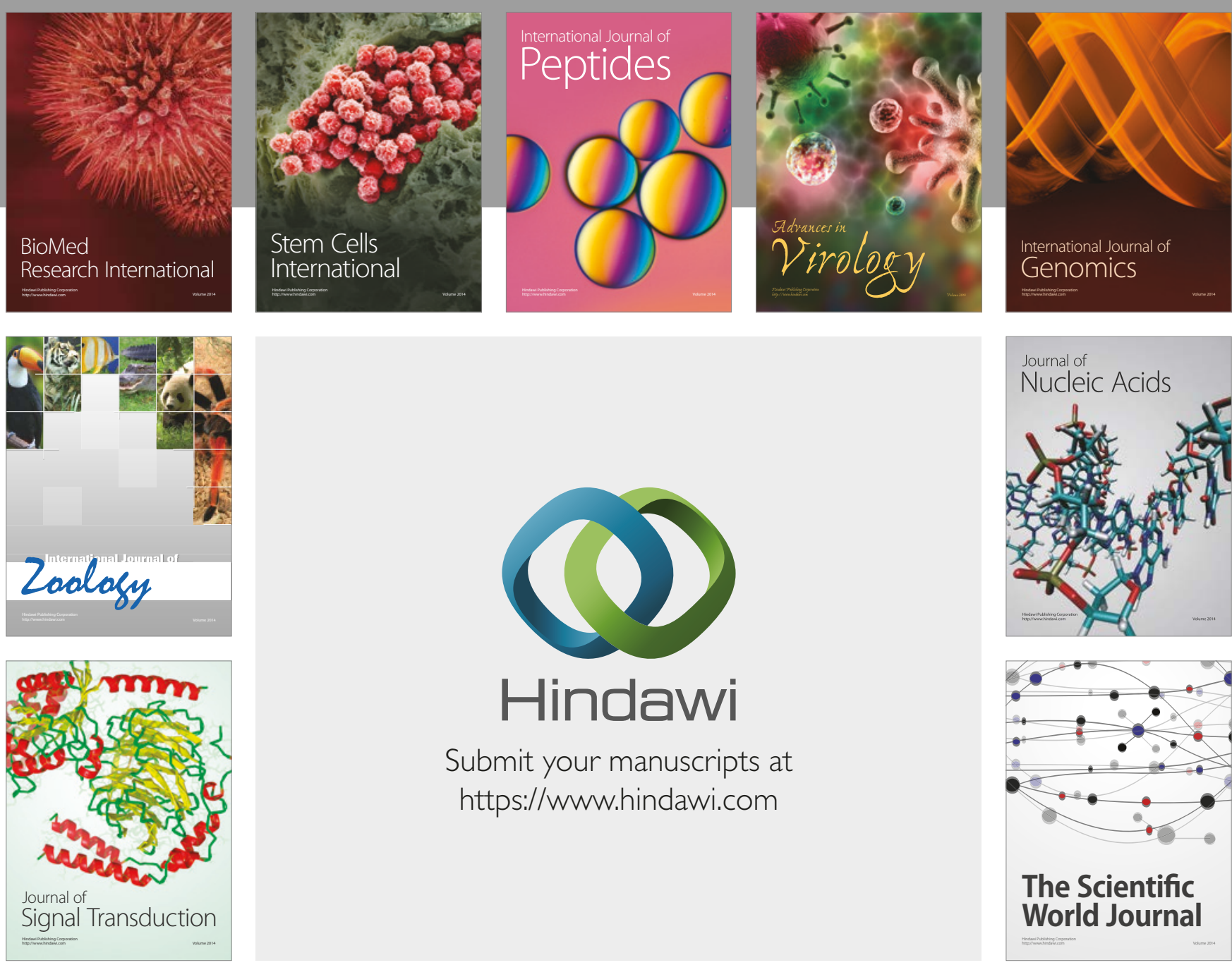

Submit your manuscripts at

https://www.hindawi.com
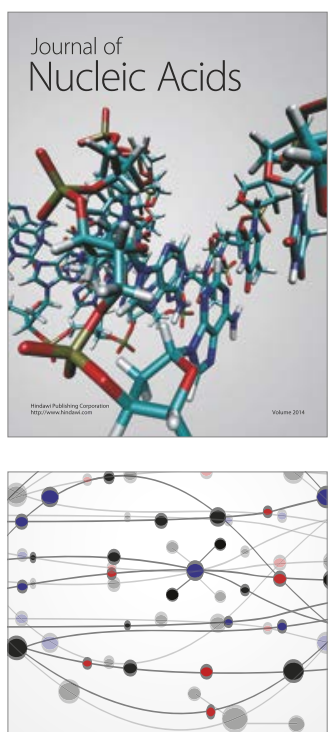

The Scientific World Journal

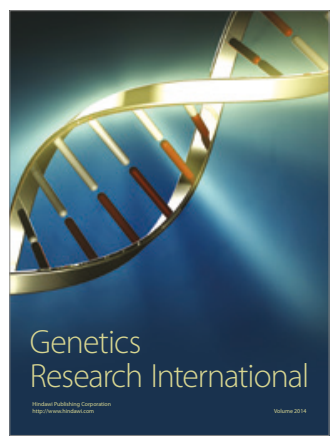

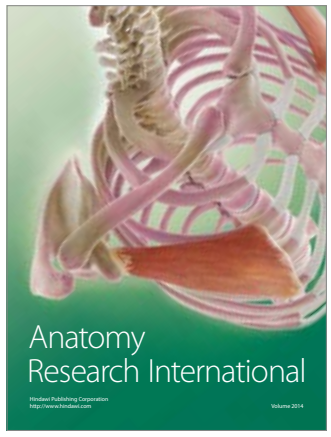

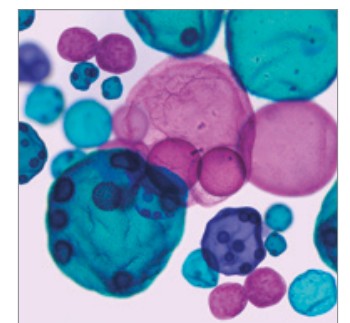

International Journal of Microbiology
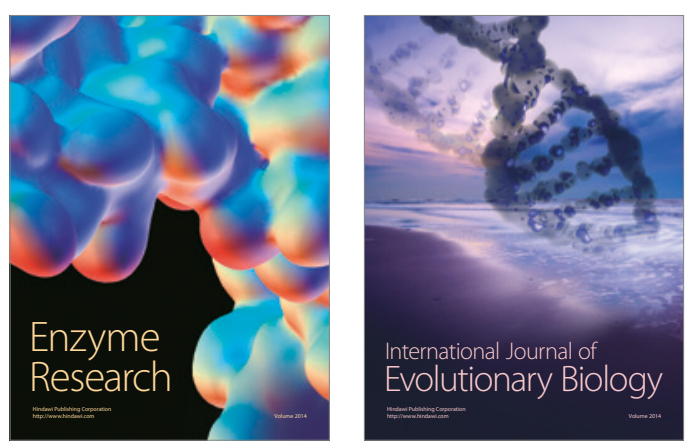
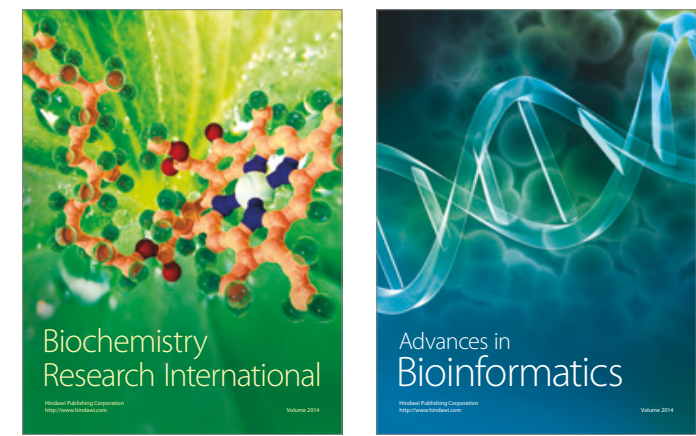

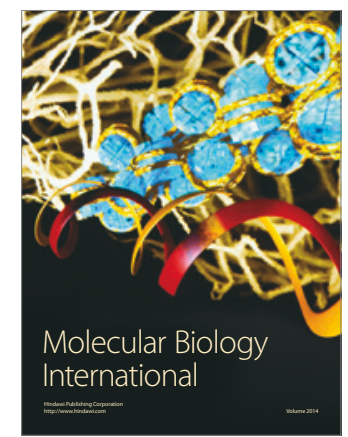

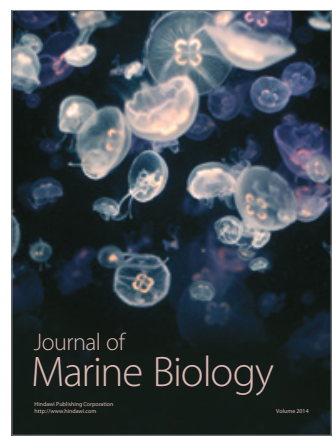

